\title{
Smoking and infarct size among STEMI patients undergoing primary angioplasty
}

\author{
Giuseppe De Luca ${ }^{\mathrm{a}, *}$, Guido Parodi ${ }^{\mathrm{b}}$, Roberto Sciagrà ${ }^{\mathrm{c}}$, Benedetta Bellandi ${ }^{\mathrm{b}}$, \\ Vincenzo Comito $^{\mathrm{b}}$, Ruben Vergara ${ }^{\mathrm{b}}$, Angela Migliorini ${ }^{\mathrm{b}}$, Renato Valenti ${ }^{\mathrm{b}}$, \\ David Antoniucci ${ }^{\mathrm{b}}$ \\ a Division of Cardiology, "Maggiore della Carità" Hospital, Eastern Piedmont University, Novara, Italy \\ ${ }^{\mathrm{b}}$ Division of Cardiology Careggi Hospital, Florence, Italy \\ ${ }^{\mathrm{c}}$ Nuclear Medicine Unity, University of Florence, Florence, Italy
}

\section{A R T I C L E I N F O}

\section{Article history:}

Received 18 May 2013

Received in revised form

30 November 2013

Accepted 8 December 2013

Available online 4 January 2014

\section{Keywords:}

Smoking

Infarct size

STEMI

Primary angioplasty

\begin{abstract}
A B S T R A C T
Background: Prior studies have found that smokers with STEMI have lower mortality rates and a more favorable response to fibrinolytic therapy than nonsmokers, phenomenon defined as "the smoker's paradox". Still poorly explored is the impact of cigarette smoking in patients undergoing primary percutaneous coronary intervention. Aim of the current study was to evaluate the impact of cigarette smoking on scintigraphic infarct size in STEMI patients undergoing primary $\mathrm{PCI}$.

Methods: Our population is represented by 830 STEMI patients undergoing primary PCI. Infarct size was evaluated at 30 days by technetium-99m-sestamibi.

Results: Smoking was associated with younger age $(p<0.001)$, a lower prevalence of female gender $(p<0.001)$, hypertension $(p<0.001)$, diabetes $(p=0.003)$, shorter ischemia time $(p=0.037)$, but higher rates of previous PCI $(p=0.016)$. No differences were observed in other clinical or angiographic characteristics. In particular, smoking did not affect the rate of postprocedural TIMI 3 flow. As shown in Fig. 1, smoking did not affect infarct size $(12.5 \%$ [3.3\%-23.7\%] vs $12.7 \%[4.9 \%-25.9 \%], p=0.12)$. Similar results were observed in subanalyses according to infarct location (anterior STEMI, $p$ int $=0.33$ ), gender ( $p$ int $=0.95)$ age, $(p$ Int $=0.96)$, diabetes $(p$ int $=0.85)$. The absence of any impact of smoking on infarct size was confirmed after correction for baseline characteristics, such as age, gender, hypertension, diabetes, previous $\mathrm{PCI}$, ischemia time (OR $[95 \% \mathrm{CI}]=0.80[0.59-1.09], p=0.15$ ).

Conclusions: This study shows that among STEMI patients undergoing primary PCI smoking status does not affect infarct size.
\end{abstract}

(c) 2014 Elsevier Ireland Ltd. All rights reserved.

\section{Introduction}

It is well know that smoking is a major risk factors for coronary artery disease (CAD), with significantly higher rates of ST-segment elevation myocardial infarction (STEMI) and death [1-3]. Paradoxically, despite the increased occurrence of STEMI in active smokers, prior studies have found that the mortality rate of smokers after STEMI is lower than in nonsmokers [4-7], especially after fibrinolytic therapy [8-12]. This phenomenon, defined as "the smoker's paradox", has been partly explained by fewer coexisting high-risk features in patients with STEMI who are current smokers [6-12]. In addition, is has been supposed a difference in lesion composition

\footnotetext{
* Corresponding author. Tel.: +390321 3733141; fax: +390321 3733407. E-mail addresses: giuseppe.deluca@maggioreosp.novara.it,p.de_luca@libero.it (G. De Luca).
}

with a greater thrombotic component and relatively less atherosclerotic plaque burden in smokers [10], thereby contributing to the more benign long-term prognosis in these patients.

The impact of cigarette smoking on clinical outcome in patients undergoing primary percutaneous coronary intervention has not been largely investigated. In particular, the supposed higher thrombotic component may be associated with impaired reperfusion and larger infarct size when a mechanical reperfusion is performed. Therefore, the aim of the current study was to evaluate whether cigarette smoking does affect scintigraphic infarct size among STEMI patients undergoing primary angioplasty.

\section{Materials and methods}

Our population is represented by 830 STEMI patients treated by primary angioplasty, who were included in randomized trials 
conducted between 2001 and 2009, that aimed at the evaluation of infarct size at 30 days after intervention [13-15]. All patients were admitted within $12 \mathrm{~h}$ from symptom onset, and received at the time of diagnosis aspirin (500 mg intravenously) and heparin (60 IU/Kg intravenously), as much as beta-blockers and nitroglycerine intravenously if not contraindicated, whereas the decision to provide glycoprotein IIb-IIIa inhibitors was left at the discretion of the operator at the time of intervention. All patients were on dual oral antiplatelet therapy (aspirin and clopidogrel or ticlopidine) for at least 4 weeks after stent implantation. All demographic, clinical, procedural and in-hospital and follow-up data were collected in a database.

\subsection{Coronary angiography and mechanical revascularization}

Selective coronary angiography was performed in multiple projections before mechanical reperfusion. Immediately after diagnostic angiography, percutaneous coronary intervention with stenting of the infarct-related vessel was performed using standard material. Successful primary percutaneous coronary intervention was defined as Thrombolysis In Myocardial Infarction (TIMI) grade 3 coronary flow in the treated vessel with a residual stenosis $<20 \%$.

\subsection{Infarct size assessment}

As previously described [15], gated single-photon emission computed tomography (SPECT) acquisition began 60 min after technetium-99m-sestamibi injection (740 MBq), using a doublehead gamma-camera equipped with high-resolution collimators, $180^{\circ}$ rotation arc, 32 projections, 60 s/projection, 8 frames/heart cycle and $64 \times 64$ matrices. The studies were reconstructed using filtered back-projection without attenuation or scatter correction and realigned along the heart axis. Perfusion defects were quantified as percentage of LV wall, with the defect threshold set at $60 \%$ of peak uptake [16].

\subsection{Statistical analysis}

Statistical analysis was performed with the SPSS 17.0 statistical package. Continuous data were expressed as median [25-75th percentiles] and categorical data as percentage. The analysis of variance test (ANOVA) or Mann-Whitney $U$ test was appropriately used for continuous variables, according to the normality of distribution, as evaluated by the Shapiro-Wilk test. The chi-square test or the Fisher's exact test was used for categorical variables. Multiple logistic regression analysis was used to evaluate the impact of smoking on infarct size after adjustment for significant $(p<0.05)$ confounding baseline characteristics. A propensity score analysis was performed in order to evaluate the impact of smoking on infarct size in homogeneous subgroups of patients.

\section{Results}

Patients' characteristics are shown in Tables 1 and 2. Smoking was associated with younger age $(p<0.001)$, a lower prevalence of female gender $(p<0.001)$, hypertension $(p<0.001)$, diabetes $(p=0.003)$, shorter ischemia time $(p=0.037)$, but higher rates of previous PCI $(p=0.016)$. No differences were observed in other clinical or angiographic characteristics. In particular, smoking did not affect the rate of postprocedural TIMI 3 flow. As shown in Fig. 1, smoking did not affect infarct size (12.5\% [3.3\%-23.7\%] vs $12.7 \%$ [4.9\%-25.9\%], $p=0.12$ ).

Similar results were observed according to tertiles of propensity score (Fig. 2) and in subanalyses according to infarct location (anterior STEMI: 9.6\% [4.1\%-25.1\%] vs 10.2\% [3.9\%-22.7\%], $p=0.34$;
Table 1

Demographic and clinical characteristics according to history of smoking.

\begin{tabular}{llll}
\hline Variable & Smoking $(n=401)$ & Control $(n=429)$ & $p$ value \\
\hline Age & $61[53-69]$ & $67[59-75]$ & $<0.001$ \\
Age $>$ 75 ys (\%) & 7.2 & 32.2 & $<0.001$ \\
Female gender (\%) & 14 & 27.3 & $<0.001$ \\
Hypertension (\%) & 33.7 & 52.9 & $<0.001$ \\
Dyslipidemia (\%) & 33.4 & 34.5 & 0.74 \\
Diabetes (\%) & 10.2 & 17.2 & 0.003 \\
Previous ACS (\%) & 5 & 3.3 & 0.21 \\
Previous CABG (\%) & 0.7 & 0.9 & 1.0 \\
Previous PTCA (\%) & 5.2 & 2.1 & 0.016 \\
Ischemia time (minutes) & $195[140-269]$ & $210[155-280]$ & 0.037 \\
Ischemia time >3 h (\%) & 55.4 & 62.9 & 0.03 \\
Anterior MI (\%) & 36.9 & 43.1 & 0.068 \\
Cardiogenic shock (\%) & 3.0 & 4.9 & 0.16 \\
\hline
\end{tabular}

non-anterior STEMI: $12.6 \%$ [3.2\%-23.2\%] vs 13.2\% [5.3\%-26.3\%], $p=0.38 ; p$ int $=0.33$ ), gender (female gender: $11.6 \%[2 \%-20.3 \%]$ vs $11 \%$ [4\%-22\%], $p=0.25$; male gender: [14.5\% [5.7\%-28.6\%] vs $16.1 \%$ $[6.0 \%-31.0 \%]], p=0.018 ; p$ int $=0.95)$ age, $(>65$ years $12.9 \%[5 \%-$ $23.8 \%$ ] vs $15.3 \%$ [6.5\%-28\%], $p=0.18 ;<65$ years: $3.6 \%$ [0-18.7\%] vs $8.6 \%[0-19 \%], p=0.28 ; p$ int $=0.96$ ), diabetes (yes: $11.9 \%[1.8 \%-$ $23.8 \%$ ] vs $12.4 \%$ [4.6\%-23.8\%], $p=0.78$; no: $12.6 \%$ [3.7\%-23.6\%] vs $15.0 \%[5.2 \%-27.4 \%], p=0.067 ; p$ int $=0.85$ ), without any significant interaction for each variable.

The absence of any impact of smoking on infarct size was confirmed when the analysis was conducted according to the percentage of patients with infarct size above the median (Fig. 3), even after correction for baseline characteristics, such as age, gender, hypertension, diabetes, previous $\mathrm{PCI}$, ischemia time (OR [95\% $\mathrm{CI}]=0.80$ [0.59-1.09], $p=0.15$ ).

\section{Discussion}

This is the largest prospective study to date evaluating the impact of cigarette smoking on infarct size among STEMI patients undergoing mechanical reperfusion. We did not find any impact of smoking on myocardial perfusion and scintigraphic infarct size.

The application of reperfusion therapies has largely contributed to the relevant reduction in mortality observed in the last decades in the treatment of STEMI. Primary angioplasty and adjunctive

Table 2

Angiographic and procedural characteristics according to history of smoking.

\begin{tabular}{lccc}
\hline Variable & Smoking $(n=401)$ & Control $(n=429)$ & $p$ value \\
\hline Collateral circulation & & & 0.6 \\
RENTROP 0 (\%) & 89.8 & 88.8 & \\
RENTROP 1 (\%) & 6.4 & 7.8 & \\
RENTROP 2 (\%) & 3.8 & 2.7 & \\
RENTROP 3 (\%) & 0 & 0.8 & 0.56 \\
Preprocedural TIMI 3 flow (\%) & 7.5 & 8.6 & 0.25 \\
IRA & & & \\
RCA (\%) & 47.4 & 43.4 & \\
CX (\%) & 15.5 & 13.3 & \\
Graft (\%) & 0 & 0.1 & \\
LAD (\%) & 36.9 & 43.1 & 0.36 \\
LM (\%) & 0 & 0.2 & 0.16 \\
Multivessel disease (\%) & 39.8 & 43.0 & 0.95 \\
Abciximab (\%) & 91.5 & 88.6 & 0.94 \\
Stenting (\%) & 100 & 98 & 0.78 \\
DES (\%) & 5.7 & 6.2 & 0.75 \\
Direct Stenting (\%) & 76 & 77.2 & 0.16 \\
Thrombectomy (\%) & 42.4 & 41 & 0.19 \\
IABP (\%) & 3.0 & 4.9 & \\
Postprocedural TIMI 3 flow (\%) & 91.0 & 93.4 & \\
\hline
\end{tabular}




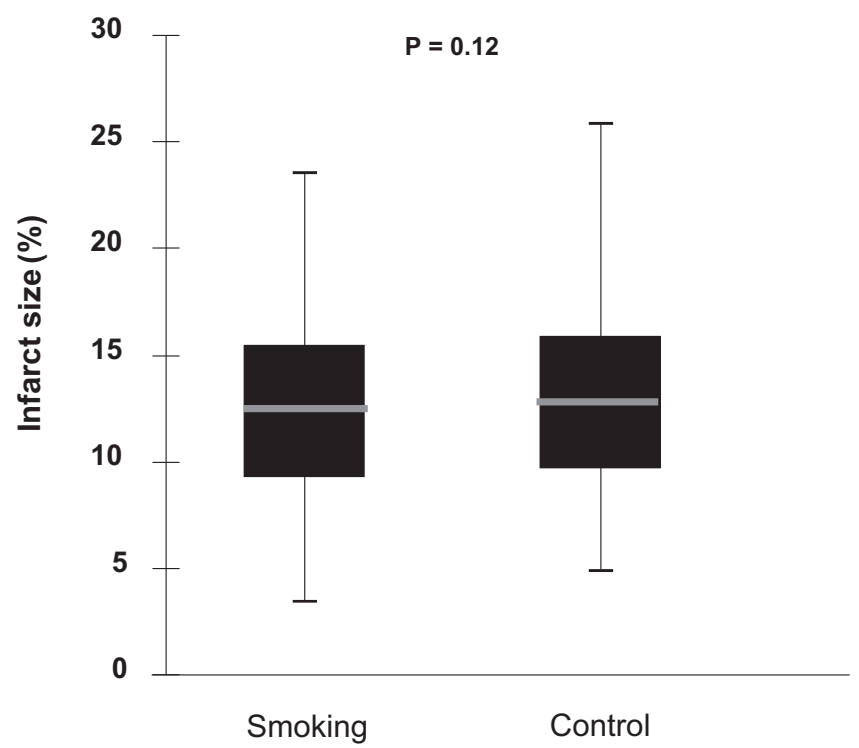

Fig. 1. Bar graphs show the impact of smoking on infarct size. Data are presented as median [25th-75th percentile].

antithrombotic therapies [17-19], have further contributed to improve survival.

Several studies have shown that smoking, despite being associated with higher occurrence of CAD and STEMI, among STEMI patients it is associated with improved survival as compared to nonsmokers, the so called "smoker's paradox". This finding has been initially observed among patients treated with thrombolysis [8-12], and some of the explanations were the lower prevalence of risk factors in smokers and the different composition of the infarctrelated lesion, with a large prevalence of thrombotic component and less atherosclerotic background. Few data have been reported among patients undergoing mechanical reperfusion, when the presence of larger thrombotic component may imply higher risk of impaired myocardial reperfusion and larger infarct size. Hasdai et al. [20] found from analysis of the GUSTO IIb study that primary angioplasty was associated with a better 30-day outcome than tissue-type plasminogen activator regardless of smoking status and should be considered when readily available. In another report, Weisz et al. [21] found that smokers had greater survival compared to nonsmokers or former smokers, both at 30 days and at 1 year of follow-up. In addition, rates of reinfarction were lower in current

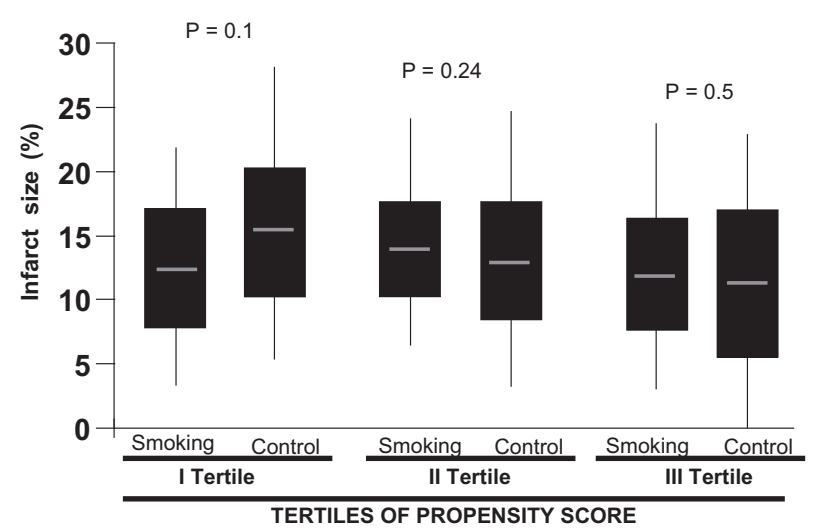

Fig. 2. Bar graphs show the impact of smoking on infarct size according to the propensity score. Data are presented as median [25th-75th percentile].

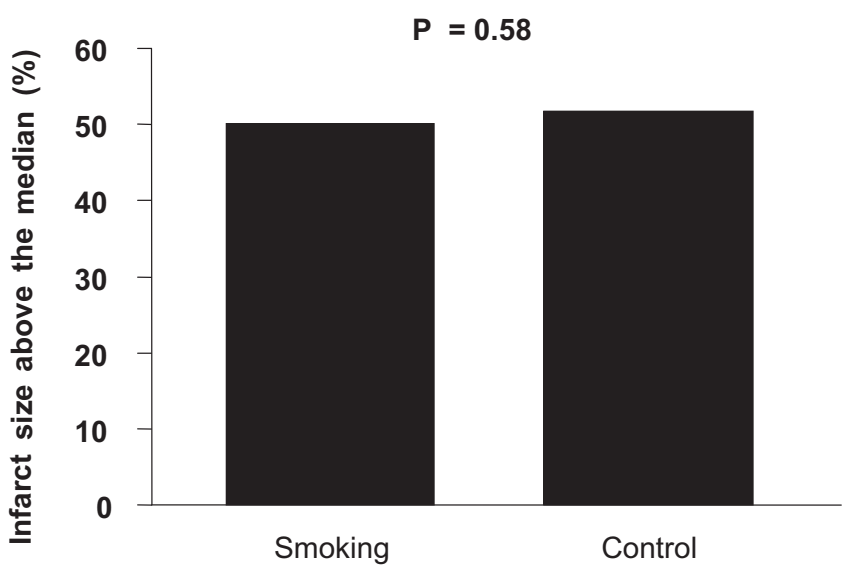

Fig. 3. Bar graphs show the impact of smoking on infarct size (as percentage of patients above the median).

smokers during the 1-year follow-up period, resulting in lower composite rates of major adverse cardiac events in cigarette users. Indeed, a dose-response curve was evident, with the greatest apparent protection from mortality and reinfarction in those who smoked the most. The improved prognosis in smokers with STEMI after primary PCI could nearly entirely be explained by differences in baseline clinical and angiographic features. Active smokers with STEMI were younger than nonsmokers and had lower rates of diabetes mellitus and hypertension, variables known to have an important impact on survival after STEMI [22,23]. Moreover, nonsmokers were more likely to be women and had higher rates of triple-vessel CAD and LAD involvement, factors also known to negatively influence prognosis after myocardial infarction [24]. When these baseline differences were corrected for by multivariate analysis, smoking status was no longer an independent predictor of survival. Similarly, in a recent study including 1424 STEMI patients undergoing rescue or primary angioplasty, Wakabayashi et al. [25], found a beneficial effect of smoking on clinical outcome, that disappeared after adjustment for differences in baseline characteristics. However, Katayama et al. [26], among 367 STEMI patients undergoing primary angioplasty, found that smoking was associated with lower mortality rates, potentially explained by less damage to the microvascular function (as evaluated by ST-segment resolution) and better ejection fraction. Similar results were found by Albertal et al. [27], who analyzed 140 STEMI patients undergoing primary angioplasty. In fact, multivariate logistic regression analysis identified active smoking as an independent predictor of complete STR at $60 \mathrm{~min}$ (OR 3.47; 95\% CI 1.48-8.14; $p=0.004$ ).

This is the largest study evaluating the impact of smoking on infarct size as evaluated by nuclear techniques. We found that smokers had a lower risk profile as compared to non-smokers. No difference was observed in scintigraphic infarct size, confirmed after correction for baseline confounding factors.

\subsection{Study limitations}

Patient reporting of smoking status may not have been accurate in all cases. Furthermore, patients were categorized as active smokers or non-smokers at the time of hospital admission, and therefore no information was collected on previous smokers. We assessed the infarct size at 1 month after index infarction instead of at hospital discharge, as in the majority of previously published studies. On the other hand, this circumstance should be more effective in preventing interference of myocardial stunning with the extent of perfusion defects [28]. Moreover, the execution of a 
coronary angiographic control before gated SPECT allowed the exclusion of infarct-related vessel restenosis. The availability of outcome data at 1 year follow-up would have improved our results Unfortunately, data were not available from all patients and therefore not included. However, this is a relatively selected cohort of patients, being included in randomized trials and 30-day survivors. Finally, we studied a patient population submitted to a very aggressive revascularisation protocol, including early direct percutaneous coronary intervention. Therefore, caution should be exercised in extending our data to infarct patients treated with other reperfusion strategies.

\section{Conclusions}

This study showed that among STEMI patients undergoing primary angioplasty, smoking does not affect scintigraphic infarct size.

\section{References}

[1] Doll R, Peto R. Mortality in relation to smoking: 20 years' observations on male British doctors. BMJ 1976;2:1525-36.

[2] Kannel WB, Higgins M. Smoking and hypertension as predictors of cardiovascular risk in population studies. J Hypertens Suppl 1990;8:S3-8.

[3] Peto R, Lopez AD, Boreham J, et al. Mortality from tobacco in developed countries: indirect estimation from national vital statistics. Lancet 1992;339: $1268-78$.

[4] Kelly TL, Gilpin E, Ahnve S, et al. Smoking status at the time of acute myocardial infarction and subsequent prognosis. Am Heart J 1985;110: $535-41$.

[5] Molstad P. First myocardial infarction in smokers. Eur Heart J 1991;12:753-9.

[6] Andrikopoulos GK, Richter DJ, Dilaveris PE, et al. In-hospital mortality of habitual cigarette smokers after acute myocardial infarction; the bsmoker's paradox Q in a countrywide study. Eur Heart J 2001;22:776-84.

[7] Gourlay SG, Rundle AC, Barron HV. Smoking and mortality following acute myocardial infarction: results from the National Registry of Myocardial Infarction 2 (NRMI 2). Nicotine Tob Res 2002;4:101-7.

[8] Barbash GI, White HD, Modan M, et al. Significance of smoking in patients receiving thrombolytic therapy for acute myocardial infarction. Experience gleaned from the International Tissue Plasminogen Activator/Streptokinase Mortality Trial. Circulation 1993;87:53-8.

[9] Barbash GI, Reiner J, White HD et al. Evaluation of paradoxic beneficial effects of smoking in patients receiving thrombolytic therapy for acute myocardial infarction: mechanism of the smoker's paradox Q from the GUSTO-I trial, with angiographic insights. Global Utilization of Streptokinase and TissuePlasminogen Activator for Occluded Coronary Arteries. J Am Coll Cardiol 1995;26:1222-9.

[10] Grines CL, Topol EJ, O'Neill WW, et al. Effect of cigarette smoking on outcome after thrombolytic therapy for myocardial infarction. Circulation 1995;91: $298-303$.

[11] Zahger D, Cercek B, Cannon CP, et al. How do smokers differ from nonsmokers in their response to thrombolysis? (the TIMI-4 trial). Am J Cardiol 1995;75: 232-6. 12.
[12] Gottlieb S, Boyko V, Zahger D, et al. Smoking and prognosis after acute myocardial infarction in the thrombolytic era (Israeli Thrombolytic National Survey). J Am Coll Cardiol 1996;28:1506-13.

[13] Antoniucci D, Migliorini A, Parodi G, et al. Abciximab-supported infarct artery stent implantation for acute myocardial infarction and long-term survival: a prospective, multicenter, randomized trial comparing infarct artery stenting plus abciximab with stenting alone. Circulation 2004;109:1704-6.

[14] Parodi G, Sciagrà R, Migliorini A, et al. A randomized trial comparing clopidogrel with ticlopidine therapy in patients undergoing infarct artery stenting for acute myocardial infarction with abciximab as adjunctive therapy. Am Heart J 2005;150:220.

[15] Migliorini A, Stabile A, Rodriguez AE, et al. Comparison of Angiojet rheolytic thrombectomy before direct infarct artery stenting with direct stenting alone in patients with acute myocardial infarction: the JETSTENT trial. J Am Coll Cardiol 2010;56:1298-306.

[16] OConnor MK, Hammel T, Gibbons RJ. In vitro validation of a simple tomographic technique for estimation of percentage myocardium at risk using methoxyisobutyl isonitrile technetium 99m (sestamibi). Eur J Nucl Med 1990; 17:69-76.

17] De Luca G, Cassetti E, Marino P. Percutaneous coronary intervention-related time delay, patient's risk profile, and survival benefits of primary angioplasty vs lytic therapy in ST-segment elevation myocardial infarction. Am Emerg Med 2009;27:712-9.

[18] De Luca G, Biondi-Zoccai G, Marino P. Transferring patients with ST-segment elevation myocardial infarction for mechanical reperfusion: a meta-regression analysis of randomized trials. Ann Emerg Med 2008;52:665-76.

[19] De Luca G, Bellandi F, Huber K, et al. Early glycoprotein IIb-IIla inhibitors in primary angioplasty-abciximab long-term results (EGYPT-ALT) cooperation: individual patient's data meta-analysis. J Thromb Haemost 2011;9:2361-70.

[20] Hasdai D, Lerman A, Rihal CS, et al. Smoking status and outcome after primary coronary angioplasty for acute myocardial infarction. Am Heart J 1999;137: 612-20.

[21] Weisz G, Cox DA, Garcia E, et al. Impact of smoking status on outcomes of primary coronary intervention for acute myocardial infarction-the smoker's paradox revisited. Am Heart J 2005;150:358-64.

[22] De Luca G, van 't Hof AW, Ottervanger JP, et al. Ageing, impaired myocardial perfusion, and mortality in patients with ST-segment elevation myocardial infarction treated by primary angioplasty. Eur Heart J 2005;26:662-6.

[23] De Luca G, Małek LA, Maciejewski P, et al. STEMI 2003 registry collaborators impact of diabetes on survival in patients with ST-segment elevation myocardial infarction treated by primary angioplasty: insights from the POLISH STEMI registry. Atherosclerosis 2010 Jun;210(2):516-20.

[24] De Luca G, Suryapranata H, van 't Hof AW, et al. Prognostic assessment of patients with acute myocardial infarction treated with primary angioplasty: implications for early discharge. Circulation 2004;109:2737-43.

[25] Wakabayashi K, Romaguera R, Laynez-Carnicero A, et al. Impact of smoking on acute phase outcomes of myocardial infarction. Coron Artery Dis 2011 Jun:22(4):217-22.

[26] Katayama T, Iwasaki Y, Yamamoto T, et al. "Smoker's paradox" in patients with acute myocardial infarction receiving primary coronary intervention J Cardiol 2006 Oct;48(4):193-200.

[27] Albertal M, Cura F, Escudero AG, et al. PREMIAR investigators. Mechanism involved in the paradoxical effects of active smoking following primary angioplasty: a subanalysis of the protection of distal embolization in high-risk patients with acute myocardial infarction trial. J Cardiovasc Med Hagerst 2008 Aug;9(8):810-2. http://dx.doi.org/10.2459/JCM.0b013e3282f73519.

[28] Sinusas AJ, Shi Q Vitols PJ, et al. Impact of regional ventricular function, geometry, and dobutamine stress on quantitative ${ }^{99 m}$ Tc-sestamibi defect size. Circulation 1993:88:2224-34. 